\title{
Time Dependent Surface Heat Transfer in Light Weight Aggregate Cement Based Materials
}

\author{
Hung Thanh Nguyen ${ }^{1,2, *}$, Frank Melandsø ${ }^{1}$, Stefan Jacobsen ${ }^{3}$ \\ ${ }^{1}$ University of Tromsø, Department of Physics, Tromsø, Norway \\ ${ }^{2}$ Narvik University College, Narvik, Norway \\ ${ }^{3}$ Norwegian University of Science and Technology, Department of Structural Engineering, Trondheim, Norway \\ E-mail:htn@hinesna.no \\ Received November 10, 2009; revised January 22, 2010; accepted January 26, 2010
}

\begin{abstract}
Surface temperature changes of building materials affect the calculation of heat flow and thus the energy use in heating and cooling. The surface heat transfer coefficient $\alpha$, limiting the heat flow between material surface and ambient air is normally taken as a constant. In this study we propose a time-dependent function $\alpha(t)$. We estimate $\alpha$ from unidirectional heat flow experiments with transient and steady-state conditions. Using temperature measurements and the conservation of energy at the surface including convective and irradiative boundary conditions, the value of $\alpha(t)$ was obtained both using Finite Difference and Taylor Polynomials methods. Numerical solutions of temperature distribution as function of time were improved with the obtained $\alpha(t)$-functions compared to with constant $\alpha$. There were no clear difference between $\alpha(t)$ on different materials, and the final values observed were in the order of magnitude expected from the literature.
\end{abstract}

Keywords: Heat Flow, Surface Heat Transfer Coefficient, Numerical Methods, Light Weight Aggregate, Cements Based Materials

\section{Introduction}

The study of heat flow properties of cement based materials (CBM) like Light Weight Aggregate (LWA) and normal weight concrete is of importance. The thermal mass of heavy building materials like brick and concrete has been found to reduce peak energy demand $[1,2]$ when properly used in a building including exposed mass facing the inside. The use of thermal mass is efficient for both cooling and heating energy demands [1-3]. In addition, heat flow properties affect structural behaviour like temperature and stress development during curing [4], moisture transport properties [5] and material durability due to for example frost exposure [6].

The better knowledge we have on models, parameters and associated data, the better we can predict heat flow, temperature rise, stress development, durability, energy use etc of varying parts of buildings and structures exposed to different temperature and moisture conditions, e.g. concrete walls and decks.

In the present investigation we LWA CBM based on some measurements of temperature distribution in LWA concrete during transient and steady-state heat flow through a surface. We have chosen to pursue the use of a time dependent surface heat transfer coefficient, $\alpha(t)$, to improve calculation of heat flow between material and surrounding air.

\section{The Surface Heat Transfer Coefficient $\alpha(t)$}

Heat flow through a building material like concrete is described by the heat equation. In one dimension with initially constant temperature and a subsequent temperature change at one end it can be written by Fourier's second law as follows $[7,8]$ :

$$
\rho C \frac{\partial T}{\partial t}=\lambda_{\text {concrete }} \frac{\partial^{2} T}{\partial x^{2}}
$$

where $T:$ Temperature $[\mathrm{K}]$ or $\left[{ }^{\circ} \mathrm{C}\right]$

$t: \quad$ time $[\mathrm{s}]$

$x: \quad$ thickness $[\mathrm{m}]$

$C: \quad$ heat capacity of material $[\mathrm{J} / \mathrm{kg} \cdot \mathrm{K}]$

$\rho: \quad$ density of material $\left[\mathrm{kg} / \mathrm{m}^{3}\right]$

$\lambda_{\text {concrete }}:$ heat conductivity of concrete $[\mathrm{W} / \mathrm{mK}=$ 
$\mathrm{J} / \mathrm{sm} \cdot \mathrm{K}]$.

where $C, \rho$ and $\alpha$ concrete are normally assumed constant in mathematical solutions of Equation (1). The heat transfer is proportional to the rate of heat flow across the solid-air interface and the temperature difference across the interface. Thus, the boundary condition may be expressed by the following Equation (7)

$$
q=\lambda_{\text {concrete }} \frac{\partial T}{\partial x}=\alpha\left(T_{\text {surf }}-T_{\text {amb }}\right)
$$

where $q:$ heat flow $\left[\mathrm{W} / \mathrm{m}^{2}\right]$

$\alpha$ : surface heat transfer coefficient $\left[\mathrm{W} / \mathrm{m}^{2} \mathrm{~K}\right]$

$T_{\text {surf }}:$ temperature at material surface $[\mathrm{K}]$

$T_{a m b}$ : temperature in ambient air adjacent to the solid-air interface [K].

The surface heat transfer coefficient for buildings materials surfaces is usually assumed to depend mainly on the convection of the air whereas heat flow by radiation and conduction is low [8]. If the control surface includes no mass or volume, the conservation of energy requirement becomes:

$$
q_{\text {cond }}-q_{\text {conv }}-q_{\text {rad }}=0
$$

This conservation requirement holds for both steadystate and transient conditions. $q_{\text {cond }}\left[\mathrm{W} / \mathrm{m}^{2}\right]$ is a conduction heat flux from the medium to the surface, $q_{c o n v}$ is convection from the surface to the fluid (air) and $q_{\mathrm{rad}}$ is net radiation exchange from the surface to the surroundings. Each of the terms may thus be described by:

$$
\lambda_{\text {concrete }} \frac{\Delta T}{\Delta x}-\alpha_{\text {conv }}\left(T_{\text {surf }}-T_{\text {amb }}\right)-\alpha_{\text {rad }}\left(T_{\text {surf }}-T_{\text {amb }}\right)=0 \text { (4) }
$$

or

$$
\lambda_{\text {concrete }} \frac{\Delta T}{\Delta x}=\alpha_{\text {tot }}\left(T_{\text {surf }}-T_{\text {amb }}\right)
$$

where $\alpha_{\text {tot }}=\alpha_{\text {conv }}+\alpha_{\text {rad }}$ is total surface heat transfer coefficient $\left[\mathrm{W} / \mathrm{m}^{2} \mathrm{~K}\right]$

$\alpha_{\text {conv }}$ : convective surface heat transfer coefficient $\left[\mathrm{W} / \mathrm{m}^{2} \mathrm{~K}\right]$

$\alpha_{\text {rad }}$ : radiation surface heat transfer coefficient $\left[\mathrm{W} / \mathrm{m}^{2} \mathrm{~K}\right]$

$$
\alpha_{\text {rad }}=\varepsilon \sigma\left(T_{\text {surf }}+T_{\text {amb }}\right)\left(T_{\text {surf }}^{2}+T_{\text {amb }}^{2}\right)
$$

where $\varepsilon$ : emissivity (no unit), maximum value = unity, typical value $0.88[8,9]$

$$
[8,9]
$$

$\sigma$ : Stefan-Boltzman's constant $=5.67 \times 10^{-8} \mathrm{~W} /\left(\mathrm{m}^{2} \mathrm{~K}^{4}\right)$

The total surface heat transfer coefficient $\alpha_{t o t}$ in Equation (5) also includes the convective surface heat transfer coefficient which comprises two mechanisms. These are energy transfer due to diffusion and due to the bulk or macroscopic motion of fluid. The convection heat transfer mode is sustained both by random molecu- lar motion and by the bulk motion of the fluid within the boundary layer. The contribution due to random molecular motion (diffusion) dominates near the surface when the fluid velocity is low. In fact, at the interface between the surface and the fluid, the fluid velocity is zero and heat is transferred by diffusion mechanism only. The contribution due to bulk fluid motion originates from the fact that the boundary layer grows as the flow progresses or penetrates in the direction normal to the surface [9]. In the rest of the paper we use the notation $\alpha$ instead of $\alpha_{t o t}$ as defined in Equation (5).

In many practical problems in fluid mechanics the heat transfer is defined as a constant. In fact, heat transfer is not a constant characteristic of the fluid medium. On the contrary, the heat transfer coefficient depends in a complicated way on many variables, including the fluid properties heat conductivity, viscosity, density, heat capacity $\left(\lambda_{f}, \mu_{f}, \rho_{f}, C_{f}\right)$, the system geometry, ambient and surface temperature of the system, flow velocity and phase change [10,11]. Fluid flow properties are typically described by characteristic quantities such as Reynolds numbers (Re), Nusselt (Nu) and GR-numbers [7,8]. Tables 1 and 2 show typical orders of magnitude for heat transfer coefficients from the literature $[9,10]$.

In [8] some expressions are proposed for the effect of wind on $\alpha_{\text {conv }}$, like Equation (7) for forced convection parallel to the material surface of a building:

$$
\begin{aligned}
& \alpha_{\text {conv }}=6+4 \mathrm{v}, \quad \mathrm{v} \leq 5 \mathrm{~m} / \mathrm{s} \\
& \alpha_{\text {conv }}=7.4 \times \mathrm{v}^{0.78}, \mathrm{v} \geq 5 \mathrm{~m} / \mathrm{s}
\end{aligned}
$$

Table 1. Typical value of the convection heat transfer coefficient $\alpha$, adapted from [9].

\begin{tabular}{ll}
\hline Process/system & $\alpha_{\text {conv }}\left[\mathrm{W} /\left(\mathrm{m}^{2} \mathrm{~K}\right)\right]$ \\
\hline Free convection & $2-25$ \\
Gases & $50-1000$ \\
Liquids & $25-250$ \\
Forced convection & $100-20000$ \\
Gases & $2500-100000$ \\
Liquids & \\
Convection with phase change & Boiling or condensation \\
\hline
\end{tabular}

Table 2. Typical orders of magnitude for heat transfer coefficient $\alpha$, adapted from [10].

\begin{tabular}{cl}
\hline Process/system & $\alpha_{\text {conv }}\left[\mathrm{W} /\left(\mathrm{m}^{2} \mathrm{~K}\right)\right]$ \\
\hline Free convection & $3-20$ \\
Gases & $100-600$ \\
Liquids & $1000-20000$ \\
Boiling water & $10-100$ \\
Forced convection & $50-500$ \\
Gases & $500-10000$ \\
Liquids & $1000-100000$ \\
Water &
\end{tabular}


As rough values $\alpha_{\text {indoor }}=6$ and $\alpha_{\text {outdoor }}=25$ can be used. The difficulties of measuring surface heat flow at very high air velocities have been discussed [12]. More recent experiments have shown that effective surface heat transfer coefficients can be in the range 40 to 140 $\mathrm{W} / \mathrm{m}^{2} \mathrm{~K}(\mathrm{~T}<273 \mathrm{~K})$ and even higher than $200 \mathrm{~W} / \mathrm{m}^{2} \mathrm{~K}(\mathrm{~T}$ $>273 \mathrm{~K})[13,14]$ at velocities around $40 \mathrm{~m} / \mathrm{s}$. Equation (7) therefore seems to give values within the right order of magnitude. The effect of temperature alone on $\alpha$ for the case of natural convection at interior surfaces is in [8] suggested as:

$$
\alpha_{\text {nat }}=2\left|T_{\text {amb }}-T_{\text {surf }}\right|^{1 / 4}
$$

Equation (8) predicts far too high temperature drops from the surface to the ambient temperature for $\alpha=6$. If the radiation is significant this will of course affect the result according to Equation (6).

Based on the above discussion the convective part of the surface heat transfer coefficient includes a time dependency due to mass transfer in the boundary layer, particularly when there is sudden temperature change at a material surface. This time dependency comes in addition to the time dependent effect on $\alpha$ of phase change as described by [11]. Phase transitions at the surface by condensation/evaporation, frosting/melting or sublimation/de-sublimation will affect the surface heat transfer due to associated heat flow. In [11] analytical models were developed and verified showing how the effective surface heat transfer coefficient varied over time depending on wind velocity, relative humidity and dew point for surface temperatures between 253 and $293 \mathrm{~K}$ when there were excessive amounts of vapour available. In the following part, an experiment is investigated and a model adapted to observed time dependency of the surface heat transfer coefficient for a case with very limited or no fluid (air) flow or surface phase transition.

\section{Measuring and Modelling the Time Dependent Surface Heat Transfer Coefficient $\alpha(t)$}

\subsection{Model Based on Temperature-Time Measurements}

The model was developed to obtain a functional relationship for $\alpha(t)$ for an experiment without excess moisture at the surface and very limited convection. Equation (2) is solved for heat flow at the surface of a material sample using measurements of temperature as function of time and sample length. The material sample is assumed exposed to unidirectional heat flow along the length axis between a cold and a warm side as shown schematically in Figure 1. Therefore, the surface boundary conditions based on Equation (2):

$$
\begin{gathered}
\left.\lambda_{\text {concrete }} \frac{\partial T}{\partial x}\right|_{x=0}=\alpha_{\text {warm }}(t)\left(T(0, t)-T_{\text {amb,warm }}(t)\right) \\
\left.\lambda_{\text {concrete }} \frac{\partial T}{\partial x}\right|_{x=L}=\alpha_{\text {cold }}(t)\left(T_{\text {amb,cold }}(t)-T(L, t)\right)
\end{gathered}
$$

where $T(x, t)$ : Specimen temperature at different positions and times

$T_{a m b}(t)$ : Ambient air temperature at the inside of the plastic foil, and

$\alpha_{\text {cold }}(t), \alpha_{\text {warm }}(t)$ : Surface heat transfer coefficient at cold and warm side

For a material with known heat conductivity, numerical values of $\alpha$ can be found using Equation (9). In order to test whether the numerical solution of $\alpha$ can be a function of time or constant, we introduce the following different boundary conditions:

$$
\begin{gathered}
\left.\lambda_{\text {concrete }} \frac{\partial T}{\partial x}\right|_{x=0}=\bar{\alpha}_{\text {warm }}\left(T(0, t)-\bar{T}_{\text {amb,warm }}\right) \\
\left.\lambda_{\text {concrete }} \frac{\partial T}{\partial x}\right|_{x=L}=\bar{\alpha}_{\text {cold }}\left(\bar{T}_{\text {amb }, \text { cold }}-T(L, t)\right)
\end{gathered}
$$

and

$$
\begin{gathered}
\left.\lambda_{\text {concrete }} \frac{\partial T}{\partial x}\right|_{x=0}=\bar{\alpha}_{\text {warm }}\left(T(0, t)-T_{\text {amb,warm }}(t)\right) \\
\left.\lambda_{\text {concrete }} \frac{\partial T}{\partial x}\right|_{x=L}=\bar{\alpha}_{\text {cold }}\left(T_{\text {amb,cold }}(t)-T(L, t)\right)
\end{gathered}
$$

where $\bar{\alpha}$ and $\bar{T}$ are constants. We will consider the numerical solutions of three different cases of the boundary conditions:

Equation (9): where both the ambient temperature and surface heat transfer coefficients are time-dependent.

Equation (10): where both the ambient temperature and surface heat transfer coefficients are constants. And,

Equation (11): where the ambient temperature is timedependent, while the surface heat transfer is constant.

\subsection{Temperature Test Set-Up}

Laboratory measurements of one-dimensional heat flow were conducted on cylindrical specimens of light weight

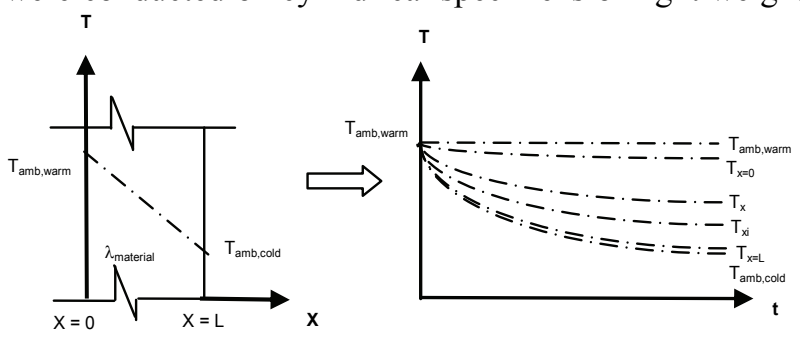

Figure 1. Principle of temperature measurements across specimen and acquired data for $\alpha(t)$. 
aggregate concrete. The test set up was made in the laboratories of Narvik University College $[15,16]$. The specimens were insulated on the cylindrical surface to ensure one-dimensional heat flow in the axial direction of the cylinder. They had $10 \mathrm{~cm}$ length and $10 \mathrm{~cm}$ diameter with thermocouples (copper-constantan) moulded along the axial direction, This was done by fixing thermocouples to a thin $(0.1 \mathrm{~mm})$ nylon thread spanning in the axial direction during moulding. The maximum aggregate size of 12-14 mm limits the accuracy of the position of the thermocouples No. 4-8 shown in Figure 2, since the accuracy of the positioning is affected by the 14 $\mathrm{mm}$ maximum aggregate size. This has been taken into account by using temperature measurements as described in Subsection 4.1. Figure 2 shows a specimen with thermocouples placed horizontally in a polystyrene pipe with $\lambda \approx 0.04 \mathrm{w} / \mathrm{mK}$. The pipe was placed in a $10 \mathrm{~cm}$ thick vertical polystyrene wall between a warm and a cold room. A $0.1 \mathrm{~mm}$ polyethylene foil covered the end of the horizontal polystyrene pipes leaving approximately $3 \mathrm{~cm}$ wide air gaps between the concrete surface and foil on warm and cold side. The role of the air gaps under the polyethylene foil is to reduce external forced convection so that it is assumed that the natural convection and radiation heat transfer acts here.

\subsection{LWA CBMs}

5 LWA CBMs were tested. The LWA consisted of spherical light weight aggregate particles produced from recycled glass raw material [17]. The particle sizes were 12-14, 10-12 and 0.3-0.6 mm, the dry particle densities were 460,480 and $770 \mathrm{~kg} / \mathrm{m}^{3}$ and the $24 \mathrm{~h}$ absorption were $13.7,19$ and $26.6 \%$ of mass. The binder of materials A, B, C and E consisted of ordinary Portland cement (CEM I) according to EN 197-1 [18], 10\% silica fume by weight of binder powder, a co-polymer water reducer (Sika viscocrete) and water was added to dry LWA to a

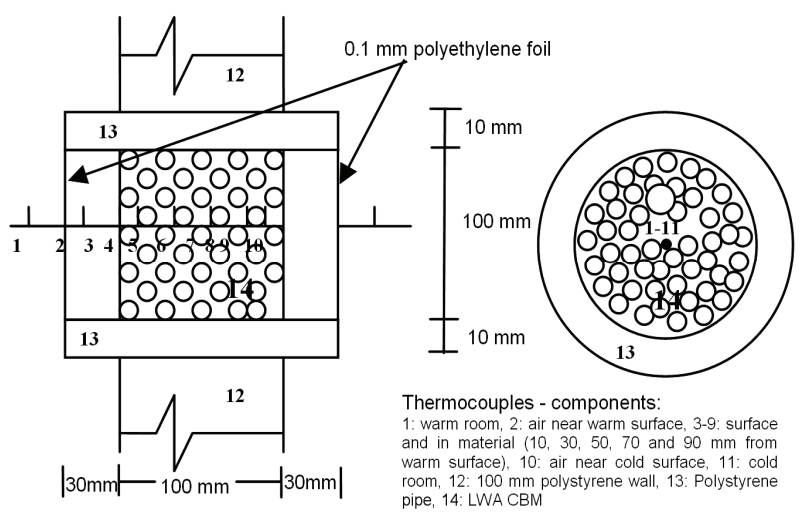

Figure 2. Side- and front view of cylindrical LWA CBM specimen in polystyrene and thermocouples along central axis (diameter $=$ length $=100 \mathbf{m m})$. total $\mathrm{w} /(\mathrm{c}+\mathrm{si})=0.45$. For Material $\mathrm{D}$ the LWA was soaked and surface dried before adding the binder (water, cement, silica fume, water reducer) to an effective $w / b=$ 0.45 so that no mix-water could be absorbed by the LWA-particles. Material A was made with LWA 12-14, Material B with 85\% 10-12 and $15 \%$ 0.3-0.6, Material C with 70\% 10-12 and 30\% 0.3-0.6 giving maximum LWA particle packing. Material D was equal to $\mathrm{C}$ but, as mentioned, made with LWA pre-soaked in water and mixed in saturated surface dry condition. Material E was made with high enough cement paste volume to fill up the void space between the individual LWA particles. Table 3 shows data of the 5 tested materials. We see that very low compressive strengths were obtained for these lowdensity materials. Further details about properties of the cement based materials and experimental set-up can be found in $[15,16,19]$.

\section{Results and Discussions}

\subsection{Temperature Measurements and Use in Modelling}

Figure 3 shows an example of measured temperature profiles for material $\mathrm{C}$ with thermocouple no.1 in warm air at the outside of the plastic foil being the upper curve at the right hand side of the plot. Thermocouple No.11 in cold air at the outside of the plastic foil is the bottom curve. The other thermocouples are in numerical order from top to bottom. Steady-state heat flow was reached in all 5 materials. The increase seen towards approximately $23^{\circ} \mathrm{C}$ at the beginning of the plot is probably due to a bit inaccurate temperature control at start of the experiment. Similar plots were made for all 5 materials but not shown here due to space considerations. Inserting the approximation of heat flux by the Finite Difference Method (FDM) as:

$$
\begin{gathered}
\left.\lambda_{\text {concrete }} \frac{\partial T}{\partial x}\right|_{x=0} \approx \lambda_{\text {concrete }} \frac{T_{6}^{j}-T_{3}^{j}}{\Delta x} \\
\left.\lambda_{\text {concrete }} \frac{\partial T}{\partial x}\right|_{x=L} \approx \lambda_{\text {concrete }} \frac{T_{9}^{j}-T_{6}^{j}}{\Delta x}
\end{gathered}
$$

in the left side of Equation (9) and rearranging we obtain

$$
\begin{gathered}
\alpha_{\text {warm }}\left(t_{j}\right)=\left(\frac{\lambda_{\text {concrete }}}{\Delta x}\right)\left(\frac{T_{6}^{j}-T_{3}^{j}}{T_{3}^{j}-T_{2}^{j}}\right) \\
\alpha_{\text {cold }}\left(t_{j}\right)=\left(\frac{\lambda_{\text {concrete }}}{\Delta x}\right)\left(\frac{T_{9}^{j}-T_{6}^{j}}{T_{10}^{j}-T_{9}^{j}}\right)
\end{gathered}
$$

where $T_{3}^{j}, T_{9}^{j}$ are the surface temperatures at time $t_{j}$ on warm and cold side respectively, $T_{6}^{j}$ is the temperature at the middle thermocouple node as illustrated in Figure 2. The ambient temperature at the warm 
Table 3. Properties of the materials.

\begin{tabular}{cccccccccc}
\hline \multirow{2}{*}{ Material } & LWA & Cement & SF & Water & W.r. & Density & $\mathrm{f}_{28}$ & $\mathrm{C}$ & $+20^{\circ} \mathrm{C}$ \\
\cline { 2 - 9 } & & & $\left(\mathrm{kg} / \mathrm{m}^{3}\right)$ & & & & $(\mathrm{MPa})$ & $\mathrm{J} / \mathrm{kg} \cdot \mathrm{K}$ & $(\mathrm{W} / \mathrm{mK})$ \\
\hline A & 211 & 136 & 15 & 68 & 0.4 & 430 & 0.5 & 896 & 0.14 \\
B & 254 & 143 & 16 & 72 & 0.5 & 486 & 0.6 & 892 & 0.15 \\
C & 319 & 155 & 17 & 78 & 0.5 & 570 & 1.1 & 888 & 0.17 \\
D & 319 & 155 & 17 & 145 & 1.0 & 637 & 1.3 & 1236 & 0.25 \\
E & 301 & 339 & 39 & 169 & 1.1 & 848 & 4.1 & 1056 & 0.23 \\
\hline
\end{tabular}

and cold sides are $T_{2}^{j}$ and $T_{10}^{j}$ respectively. The length between the surface of both sides and middle node number six is denoted $\Delta x$. The heat transfer in Equation (13) may have positive or negative value depending on the accuracy of the positioning of the thermocouples. Heat flux through the material is calculated from $\left(T_{6}^{j}-T_{3}^{j}\right)$, $\left(T_{9}^{j}-T_{6}^{j}\right)$. And, the convection terms are calculated from $\left(T_{3}^{j}-T_{2}^{j}\right),\left(T_{10}^{j}-T_{9}^{j}\right)$. In this study we consider only time steps with positive heat transfers and, therefore, it is required that

$$
T_{2}^{j}>T_{3}^{j}>T_{6}^{j} \text { and } T_{6}^{j}>T_{9}^{j}>T_{10}^{j} \text {, for } \forall j
$$

This was implemented in all calculations of $\alpha(t)$ since there were some inaccuracies in the positioning of the closest thermocouples at the surface due to the maximum LWA size of $14 \mathrm{~mm}$. We estimate the inaccuracy to be approximately $+/-7 \mathrm{~mm}$ and we therefore chose to use the above temperature measurement positions. In Figures $\mathbf{4}$ and $\mathbf{5}$ we have plotted the relevant temperatures and we see that the conditions of Equation (14) are satisfied for the warm side when $t \geq 13800 \mathrm{~s}$ and for the cold side when $t \geq 4200 \mathrm{~s}$. For that reason we did not plot the heat transfer for $\mathrm{t}=0$.

The dimensionless parameter, the Biot number [7] is defined as:

$$
\begin{aligned}
& B i_{\text {warm }}\left(t_{j}\right)=\left(\frac{T_{6}^{j}-T_{3}^{j}}{T_{3}^{j}-T_{2}^{j}}\right)=\frac{\alpha_{\text {warm }} \Delta x}{\lambda_{\text {concrete }}} \\
& B i_{\text {cold }}\left(t_{j}\right)=\left(\frac{T_{9}^{j}-T_{6}^{j}}{T_{10}^{j}-T_{9}^{j}}\right)=\frac{\alpha_{\text {cold }} \Delta x}{\lambda_{\text {concrete }}}
\end{aligned}
$$

Plots of Biot numbers are presented in Figure 6 of material $\mathrm{C}$ showing that stable values are reached after around 5 hours.

$$
\text { The quantities }\left(\frac{T_{6}^{j}-T_{3}^{j}}{T_{3}^{j}-T_{2}^{j}}\right) \text { and }\left(\frac{T_{9}^{j}-T_{6}^{j}}{T_{10}^{j}-T_{9}^{j}}\right)
$$

appearing in Equation (15) play a fundamental role in conduction problems that involve surface convection effects. The Biot number provides a measure of the temperature drop in the solid relative to the temperature difference between the surface and fluid. In a 1-D problem, for $B i \prec \prec 1$, the temperature gradient in the solid is small and $T(x, t) \approx T(t)$. In other words, it is reasonable to

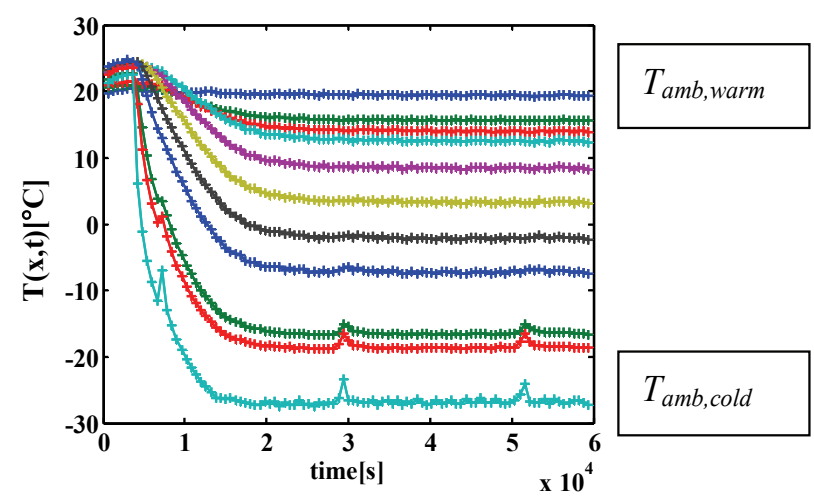

Figure 3. Measured temperatures material $\mathbf{C}$, thermocouples 1-11 from top to bottom (see Figure 2).

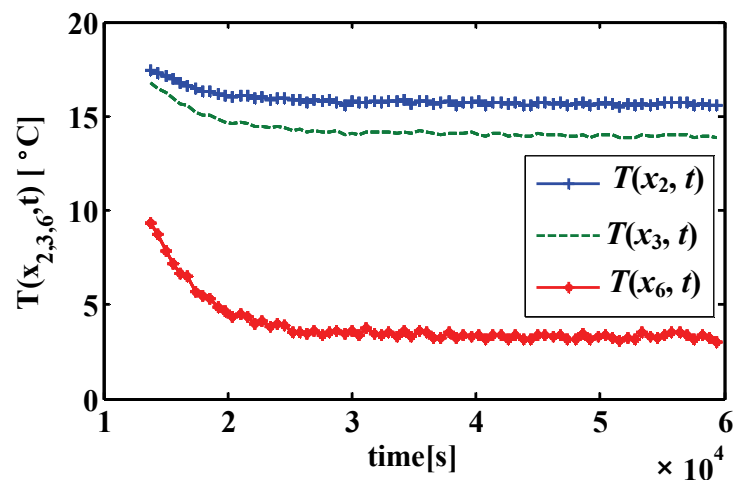

Figure 4. Temperature at position $x_{2}, x_{3}$ and $x_{6}$ for material C, with start time $t_{0}=13800 \mathrm{~s}$.

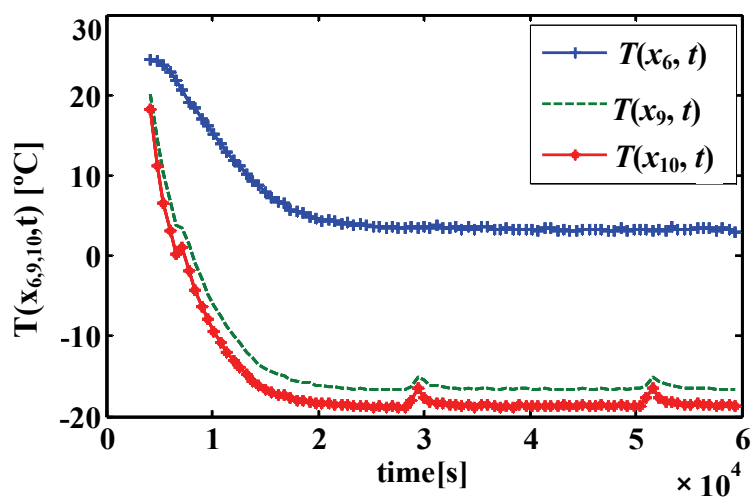

Figure 5. Temperature at position $x_{6}, x_{9}$ and $x_{10}$ for material C, with start time $t_{0}=4200 \mathrm{~s}$. 


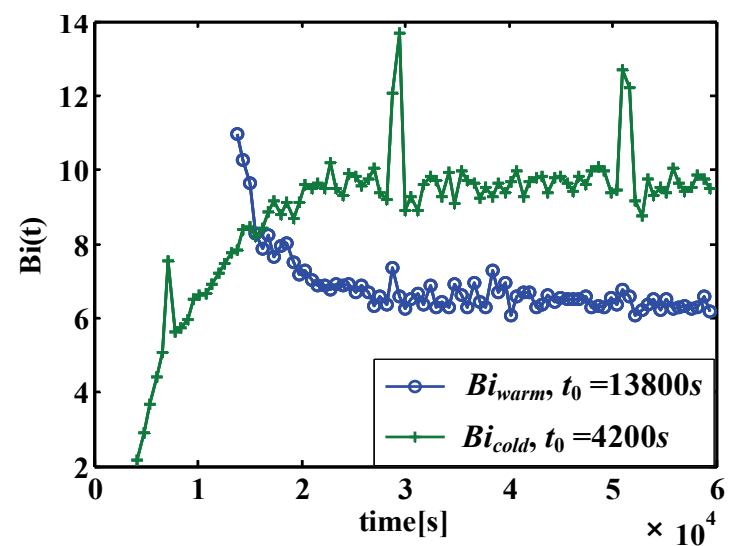

Figure 6. Biot number on warm and cold side of material $C$.

assume a uniform temperature distribution across a solid at any time during a transient process. For moderate to large values of the Biot number, however, the temperature gradients within the solid are significant. Hence, $T=T(x, t)$. Note that for $B i \succ \succ 1$, the temperature difference across the solid is much larger than that between the surface and the fluid. Furthermore, in order to calculate the heat transfer at the time $t_{j}$, then Equation (13) use temperature measured at this time. These equations did not include the time history of temperature in heat transfer calculations. A time series method has been used for this purpose [20]. To include temperature time history to determinate heat transfer coefficient, we used Taylor polynomials method (TLM). Inserting Equation (15) in Equation (13), the heat transfer at the time $t_{j}$ may be expressed by the Biot-number parameter as:

$$
\begin{aligned}
& \alpha_{\text {warm }}\left(t_{j}\right)=\left(\frac{\lambda_{\text {concrete }}}{\Delta x}\right) B i_{\text {warm }}\left(t_{j}\right) \\
& \alpha_{\text {cold }}\left(t_{j}\right)=\left(\frac{\lambda_{\text {concrete }}}{\Delta x}\right) B i_{\text {cold }}\left(t_{j}\right)
\end{aligned}
$$

Moreover, that it is a reasonable and relevant relation between the Biot-number and the heat transfer since the Biot number provides a measure of the temperature drop in the solid relative to the temperature difference between the surface and the fluid. The value of heat transfer in the next time step $\left(t_{j}+\Delta t=t_{j+1}\right)$ may be expressed by the following equation:

$$
\begin{gathered}
\alpha_{\text {warm }}\left(t_{j}+\Delta t\right) \approx \alpha_{\text {warm }}\left(t_{j}\right)+\left(\alpha_{\text {warm }}\right)^{\prime}\left(t_{j}\right) \Delta t \\
\alpha_{\text {cold }}\left(t_{j}+\Delta t\right) \approx \alpha_{\text {cold }}\left(t_{j}\right)+\left(\alpha_{\text {cold }}\right)^{\prime}\left(t_{j}\right) \Delta t
\end{gathered}
$$

where $(\alpha)^{\prime}=(\partial \alpha / \partial t)$. The foregoing Equations (17) is results of using the first order of the Taylor polynomial to approximate heat transfer function at the time $t_{j+1}$. Now, we approximate the derivation term $(\alpha)^{\prime}$ in Equa- tion (17) as:

$$
(\alpha)^{\prime}\left(t_{j}\right) \approx \frac{\alpha\left(t_{j+1}\right)-\alpha\left(t_{j-1}\right)}{2 \Delta t}
$$

Inserting Equation (18) and Equation (16) in Equation (17) and rearranging, we obtain:

$$
\begin{gathered}
\alpha_{\text {warm }}\left(t_{1}\right)=\alpha_{\text {warm }, 1} ; \alpha_{\text {cold }}\left(t_{1}\right)=\alpha_{\text {cold }, 1} \\
\alpha_{\text {warm }}\left(t_{2}\right)=\alpha_{\text {warm }, 2} ; \alpha_{\text {cold }}\left(t_{2}\right)=\alpha_{\text {cold }, 2} \\
\alpha_{\text {warm }}\left(t_{j+1}\right)=\alpha_{\text {warm }}\left(t_{j}\right)+k \frac{\left(B i_{\text {warm }}\left(t_{j+1}\right)-B i_{\text {warm }}\left(t_{j-1}\right)\right)}{2}(19) \\
\alpha_{\text {cold }}\left(t_{j+1}\right)=\alpha_{\text {cold }}\left(t_{j}\right)+k \frac{\left(B i_{\text {cold }}\left(t_{j+1}\right)-B i_{\text {cold }}\left(t_{j-1}\right)\right)}{2} \\
j=2,3, \ldots
\end{gathered}
$$

where $k$ is a constant, $k=\lambda_{\text {concrete }} / \Delta x \in R$ and $\left(\alpha_{\text {warm }, 1}, \alpha_{\text {cold }, 1}\right) ;\left(\alpha_{\text {warm }, 2}, \alpha_{\text {cold }, 2}\right)$, are start values of the surface heat transfer coefficients on warm and cold side, respectively. We note that Equation (19) use different temperature data in the time steps $t_{j-1}$ and $t_{j}$ to calculate the surface heat transfer coefficients at the time $t_{j+1}$.

\subsection{Time dependent Surface Heat Transfer, $\alpha(t)$, from Experiments}

Figures 7-12 show plots of the time dependencies of $\alpha$, $\alpha_{\text {rad }}$ and $\left(\alpha_{\text {nat }}=\alpha-\alpha_{\text {rad }}\right)$ at warm and cold side surface of material $\mathrm{C}$ and $\mathrm{A}$ respectively. The total surface heat transfer coefficients $\alpha$ are calculated with Equation (13) with temperatures measured as discussed above and shown in Figures 4 and 5. The results of $\alpha_{\text {rad }}$ are based on Equation (6). The time step used to calculate the heat transfer is in each 10 minute or 600 second interval. It is the same time step as the temperature measurements.

The start times for calculation vary in accordance with the discussion above and Equations (13) with the condition in Equations (14). It seems from figures that $\alpha(t)$ approaches, or fluctuates around, a constant value at the end of the experiment when steady state is reached like in [21]. The final values are in the range 20-35 in agreement with the values in Tables $\mathbf{1}$ and $\mathbf{2}$ and without any clear difference between warm and cold side.

In the launched test set-up we regard that heat transfer at the surface includes natural convection and radiation terms. This assumption can be made because the convection takes place only within the $3 \mathrm{~cm}$ air gaps between the polyethylene foil and the building material surface (thermocouples 2 and 10 in Figure 2). The air in the room outside the foil (thermocouples 1 and 11 in Figure 2) was cooled down with a powerful cooling machine not acting directly on the material surface due to the foil. 


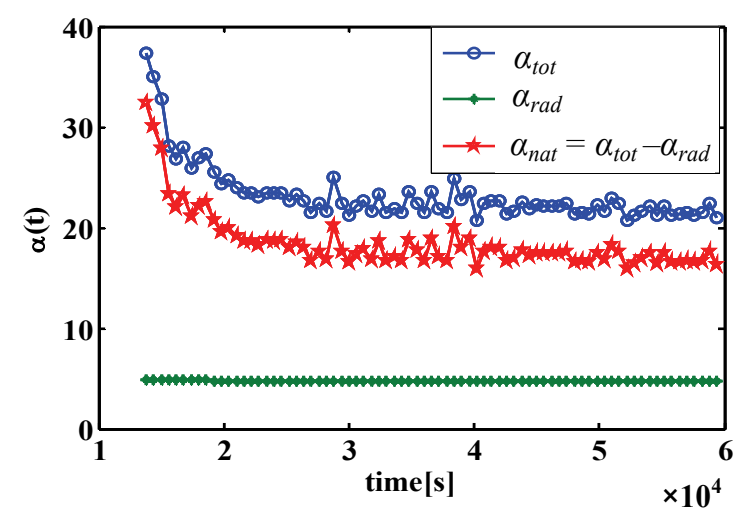

Figure 7. Total heat transfer $\alpha_{\text {warm }}(t)$ with in addition two mechanisms which are natural convection part $\alpha_{n a t}$ and radiation part $\alpha_{\text {rad }}$, of material $\mathrm{C}$, where calculation starts time $t_{0}=13800 \mathrm{~s}$.

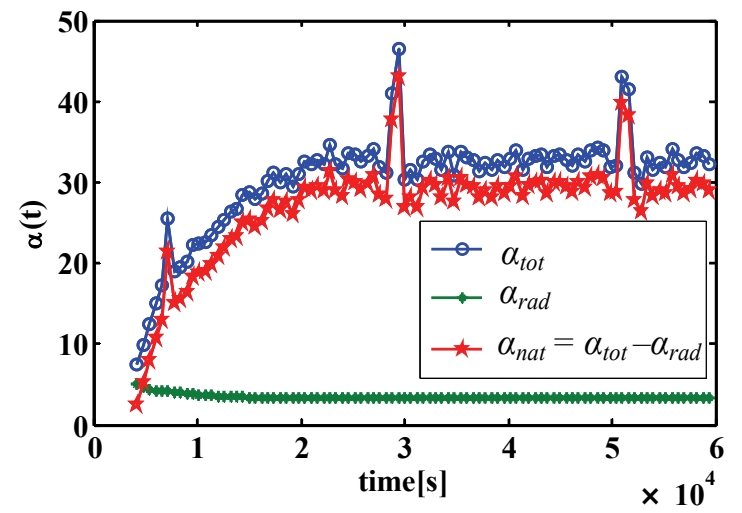

Figure 8. Total heat transfer $\alpha_{\text {cold }}(t)$ with in addition two mechanisms which are natural convection part $\alpha_{\text {nat }}$ and radiation part $\alpha_{\text {rad }}$, of material $\mathrm{C}$, where calculation starts time $t_{0}=4200 \mathrm{~s}$.

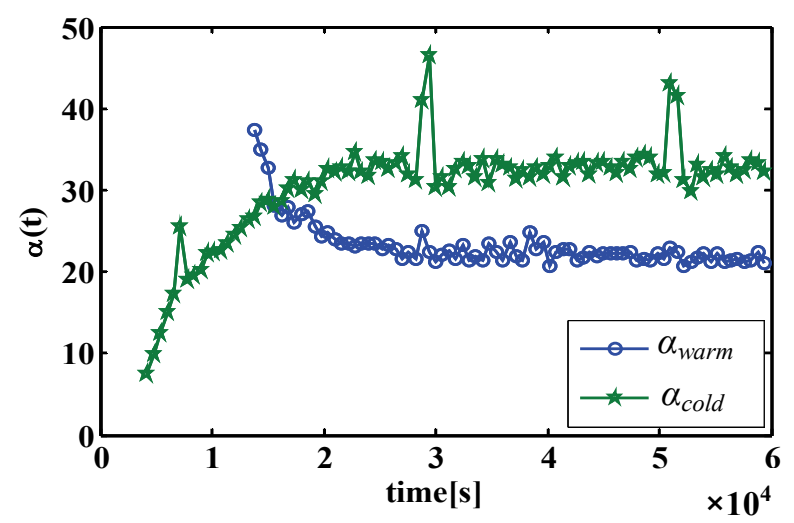

Figure 9. $\alpha_{\text {warm }}(t)$ and $\alpha_{\text {cold }}(t)$ of material $\mathrm{C}$ with different calculation of start time.

Furthermore, we disregard any effect of phase change by condensing/freezing vapour on the material surface due to the very low amount of vapour in the $3 \mathrm{~cm}$ air gaps between material surface and polyethylene foil. This is

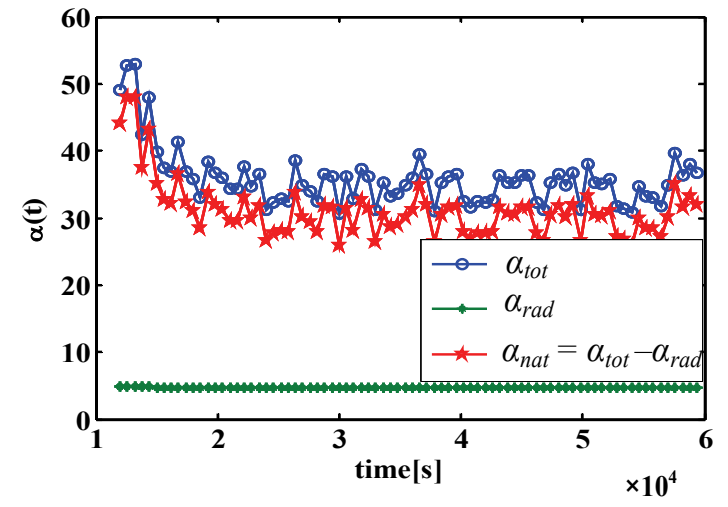

Figure 10. $\alpha_{\text {warm }}(t)$ of material A, where start time $t_{0}=$ $12000 \mathrm{~s}$.

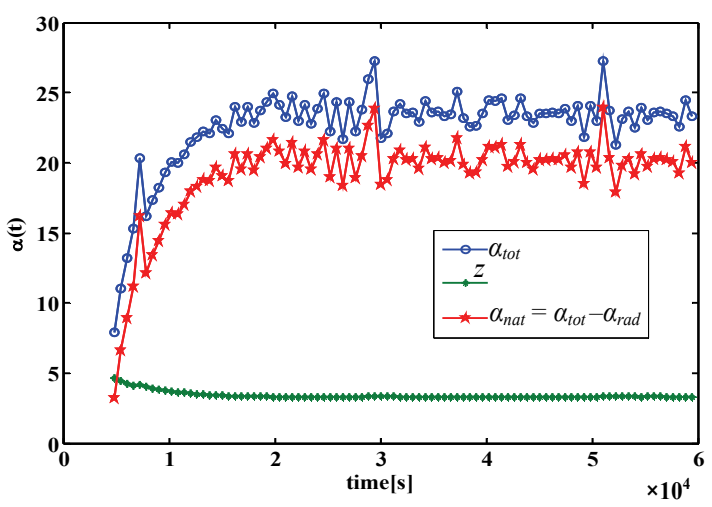

Figure 11. $\alpha_{\text {cold }}(t)$ of material A, where start time $t_{0}=4800 \mathrm{~s}$.

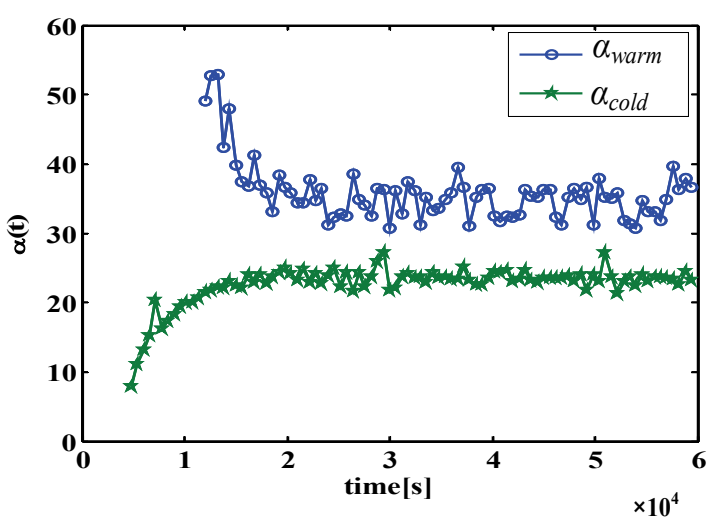

Figure 12. $\alpha_{\text {warm }}(t)$ and $\alpha_{\text {cold }}(t)$ of material A.

also supported by the low values we obtain for $\alpha$ compared to the values with phase change in Tables $\mathbf{1}$ and $\mathbf{2}$.

The radiation terms may be ignored for moderate to large convection surface heat transfer coefficients [9]. However, for natural convection in a gas, radiation may be significant and it should be included. The calculated radiation terms contributions in our experiments are shown in Figure 7.

Figure 6 shows the Biot number at the cold and warm 
side for material C. As can be observed from this figure, in the transient time period, the Biot-number at the cold side is an increasing function of time, while it is a decreasing function of time at the warm side. These Biot numbers converged to a constant value after six and a half hours. The values of the Biot numbers for both sides are larger than 1 most of the time. Based on the definition of the Biot- number in Equation (15), this means that when $B i \succ 1$ the temperature difference across the solid is much lager than between the surface and the fluid. The results agree with this argument. Figure 13 illustrates this by showing the heat flux from the surface on the cold side and temperature difference between surface and the fluid for material $\mathrm{C}$. The calculation shows that the temperature gradients within the solid are significant under the transient time period. Hence, $T=T(x, t)$.

Figures 14 and $\mathbf{1 5}$ show the surface heat transfer coefficient for two different methods; as FDM, Equation (13) and TLM, Equation (19). It seems that the resulting surface heat transfer coefficients for both models were almost equal.

Finally, in order to perform numerical simulations with the commercial FEM software [22], we need to approximate the discrete value of the time dependent surface heat transfer as shown for material C in Figure 9, with the continuous functions. For this purpose we adapted the continuous exponential function:

$$
\alpha(t)=\left(e^{-b t}\left(\alpha_{0}-\alpha_{\infty}\right)\right)+\alpha_{\infty}
$$

where $t$ : time

$$
\begin{aligned}
& b: \text { constant } \\
& \alpha(t) \rightarrow \alpha_{\infty} \text { when } t \rightarrow \infty \\
& \alpha(t) \rightarrow \alpha_{0} \text { when } t \rightarrow 0 .
\end{aligned}
$$

If $\alpha_{0}$ and $\alpha_{\infty}$ are known, then the unknown parameter

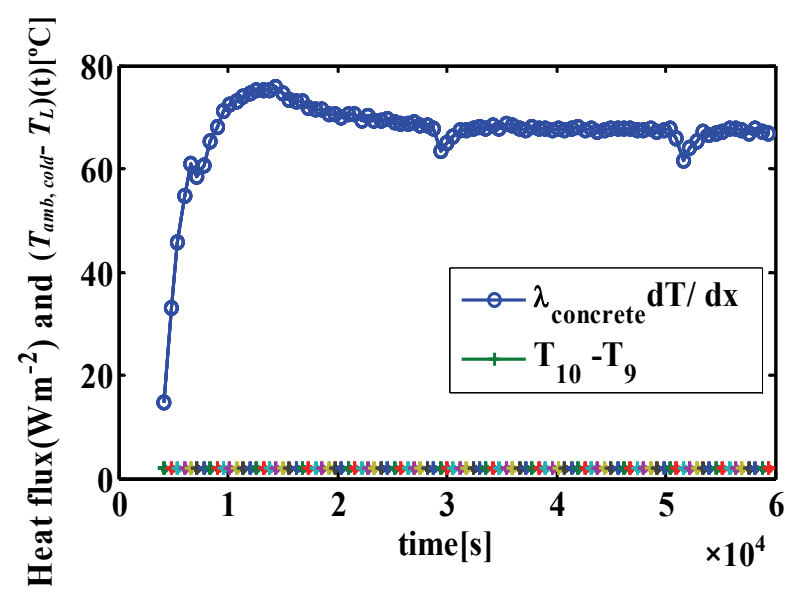

Figure 13. Heat flux at the surface and the temperature difference between the surface and the fluid at the cold side for material $\mathbf{C}$.

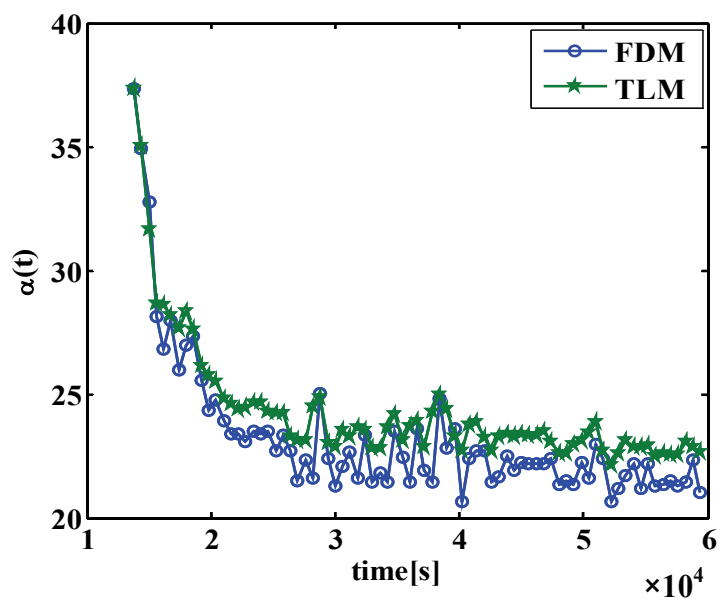

Figure 14. $\alpha_{\text {warm }}$ by Finite Difference Method (Equation (13)) and Taylor Polynomial Method (Equation (19)) for material C.

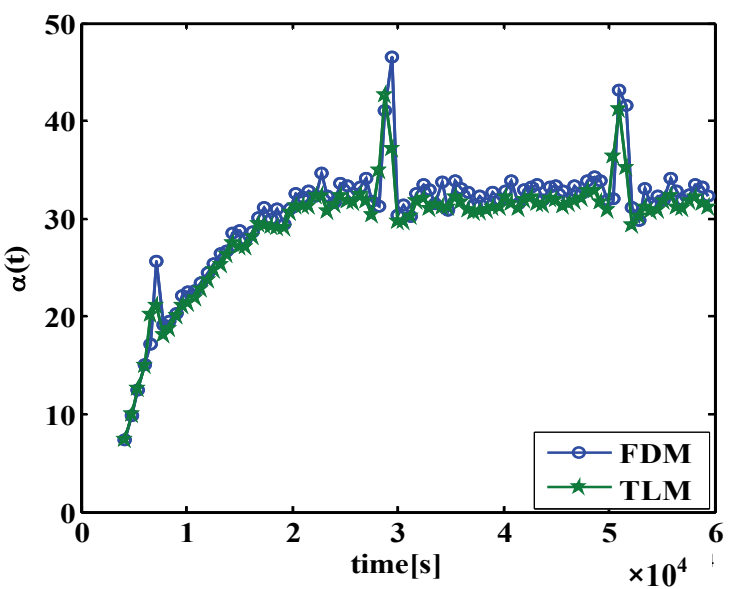

Figure 15. $\alpha_{\text {cold }}$ by Finite Difference Method (Equation (13)) and Taylor Polynomial Method (Equation (19)) for material C.

b may be solved by rearranging Equation (20):

$$
b=-\frac{1}{t_{j}} \ln \left(\frac{\alpha_{j}-\alpha_{\infty}}{\alpha_{0}-\alpha_{\infty}}\right)
$$

Where $\alpha_{j}=\alpha\left(t_{j}\right), t_{j} \in\left(t_{0}, t_{N}\right)$ and $t_{N}$ is the total time of the experiment. In our case, for material $\mathrm{C}$, from the experiments we estimate that $\alpha_{\text {cold }, 0}=0$ and $\alpha_{\text {warm }, 0}=55$, see Figures 7-12 or Table 4. In Table 4, the calculated parameters of $\alpha(t)$ from the experiments are given. $\alpha_{\infty}$ seems to reach reasonable values after a few hours, starting from the estimated $\alpha_{0}$-value on warm and cold sides. The $\alpha_{\infty}$ reached for example for material C roughly 22 and $32 \mathrm{~W} / \mathrm{m}^{2} \mathrm{~K}$ at warm and cold side respectively, and with no clear difference neither with 
Table 4. Parameters of functions for time-dependent surface heat transfer coefficient $\alpha(t)$.

\begin{tabular}{ccccccc}
\hline Material & \multicolumn{3}{c}{$\alpha_{\text {cold }}(t)$} & \multicolumn{3}{c}{$\alpha_{\text {warm }}(t)$} \\
\hline & $\mathrm{b}$ & $\alpha_{0}$ & $\alpha_{\infty}$ & $\mathrm{b}$ & $\alpha_{0}$ & $\alpha_{\infty}$ \\
$\mathrm{A}$ & $2.19 \cdot 10^{-4}$ & 0 & 23.28 & $8.96 .10^{-5}$ & 55 & 38.0 \\
$\mathrm{~B}$ & $1.95 \cdot 10^{-4}$ & 0 & 33.64 & $2.60 \cdot 10^{-4}$ & 55 & 21.04 \\
$\mathrm{C}$ & $1.90 \cdot 10^{-4}$ & 0 & 32.26 & $1.23 \cdot 10^{-4}$ & 55 & 21.03 \\
$\mathrm{D}$ & $2.53 \cdot 10^{-4}$ & 0 & 25.21 & $\mathrm{x}$ & $\mathrm{x}$ & $\mathrm{x}$ \\
$\mathrm{E}$ & $\mathrm{x}$ & $\mathrm{x}$ & $\mathrm{x}$ & $\mathrm{x}$ & $\mathrm{x}$ & $\mathrm{x}$ \\
\hline
\end{tabular}

$\mathrm{X}$ : parameters not calculated due to too large scatter of data.

varying material nor temperature. This is analogous to using the same kind of function as in Equation (20) to approximate the ambient temperature for the purpose of numerical simulation.

\subsection{Applying $\alpha(t)$ in Numerical Solutions of the Heat Flow Equation}

Finally, we investigate the effect of the obtained time dependent surface heat transfer coefficients on numerical simulations of temperature-time lapse in the material specimens. We do this by applying Equation (20) when solving the heat flow equation, Equation (1), with the boundary condition given in Equation (9). We also solve Equation (1) with constant surface heat transfer $\bar{\alpha}$ as defined in Equation (10) and (11) with constant and measured surface temperatures, respectively. We compare the solutions with the measured temperature profiles. A commercial finite element method based software $[22,23]$ was used. The software can easily be adapted to various moisture and heat flow problems in building materials $[5,14,16,22,23]$.

Figures 16-21 show the varying numerical solutions for material $\mathrm{C}$. Each figure contains four temperature-time graphs for the points $x_{3}$ and $x_{9}$ (surface), and $x_{4}, x_{6}, x_{8}$ (interior). The positions are shown in Figure 2. In addition to the measured temperature-time-graphs there are three calculated graphs. These are respectively; a time dependent $\alpha(t)$ and constant temperature according to Equation (9), constant $\bar{\alpha}$ and $\bar{T}$-values according to Equation (10), and using constant $\bar{\alpha}$-value, but measured temperature $T$, according to Equation (11). The constant value was taken as $\bar{\alpha}=25$ (see Figure 9), whereas the constant temperature at the warm side $\bar{T}_{\text {amb,warm }}=\lim _{t \rightarrow \infty} T_{a m b, \text { warm }}(t)=13.9^{\circ} \mathrm{C}$ and at the cold side $\bar{T}_{\text {amb,cold }}=\lim _{t \rightarrow \infty} T_{a m b, \text { cold }}(t)=-16.6^{\circ} \mathrm{C}$ for material C. And, the constant start-temperature of the numerical results was approximated as $T\left(x_{i}, t=0\right)=23^{\circ} \mathrm{C}$ (see temperature-time measurements in Figure 3).

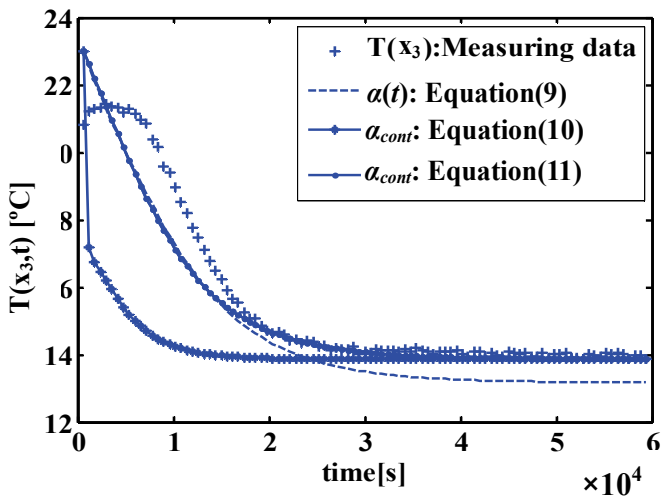

Figure 16. Numerical solution and measured temperature at the surface on the warm side $\left(x_{3}\right)$ of material $C$.

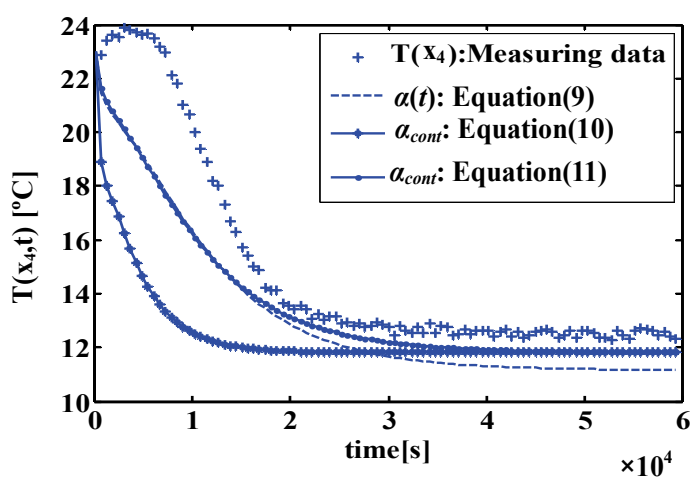

Figure 17. Numerical solution and measured temperature $\left(x_{4}\right)$ at the surface on the cold side of material $C$.

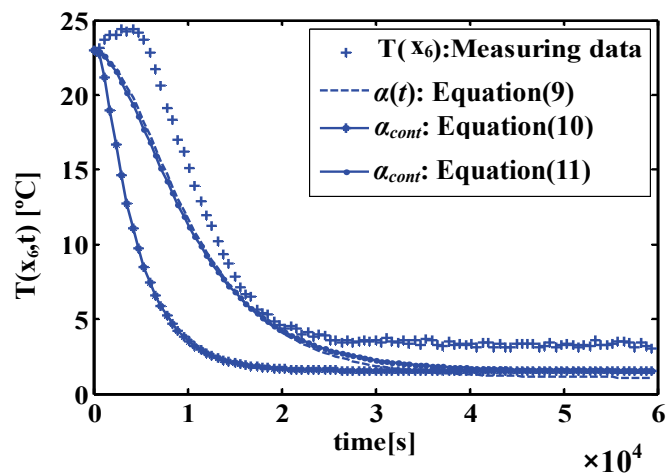

Figure18. Numerical solution and measured temperature $\left(x_{6}\right)$ at the surface on the cold side of material $C$. 


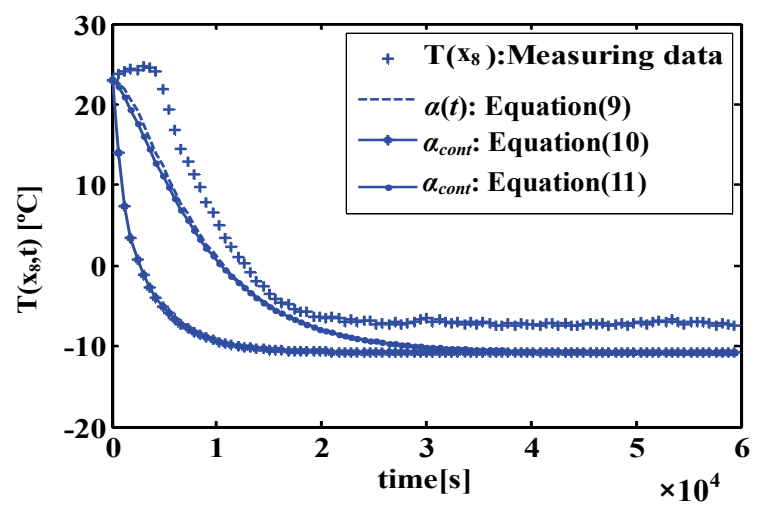

Figure 19. Numerical solution and measured temperature $\left(x_{8}\right)$ near the surface on the cold side of material $C$.

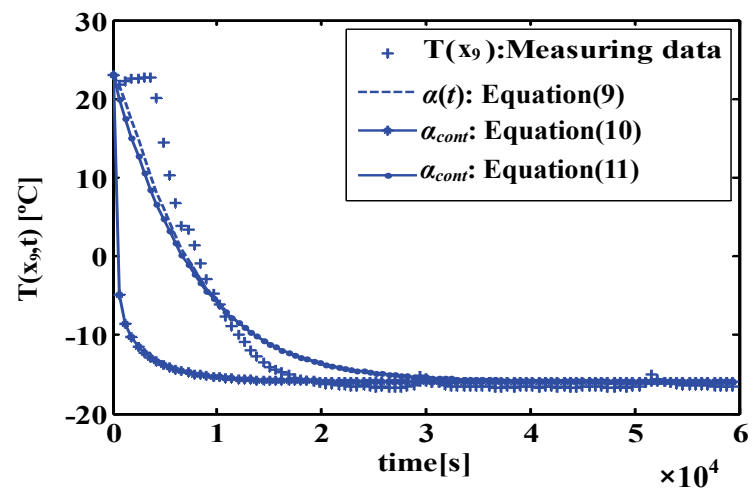

Figure 20. Numerical solution and measured temperature $\left(x_{9}\right)$ at the surface on the cold side of material $\mathrm{C}$.

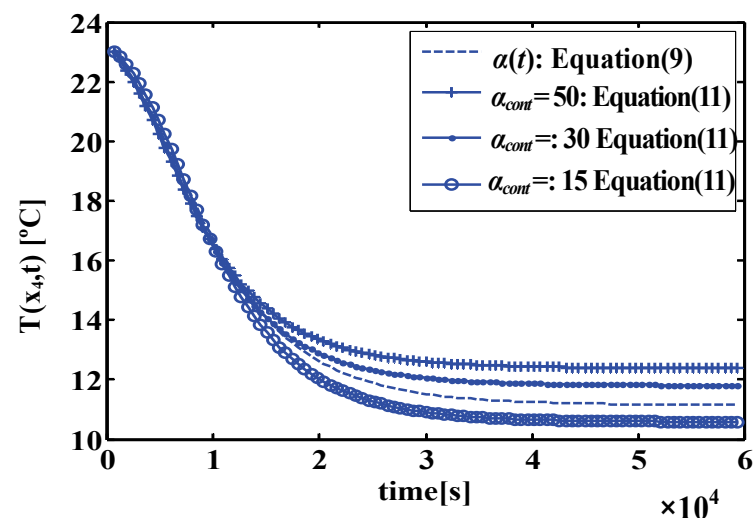

Figure 21. Numerical solution at $\left(x_{4}\right)$ of material $\mathrm{C}$ where $\alpha(t)$ in Equation (9) and different select constant heat transfer in Equation (11).

Figures 16-20 show that the time dependent heat transfer solution in Equation (9) is always a better approximation to the measured data than when using constant $\bar{\alpha}$ and constant ambient air temperature in Equation (10). The constant $\bar{\alpha}$ and the constantly low or high $\bar{T}$ in Equation (10) give far too fast temperature reduction during the unsteady-state regime compared to measured tem- peratures. However, for the two simulations with measured surface temperature (Equations (9) and (11)) we see that the resulting temperature profiles seem to coincide with the measurements and that time dependent (Equation (9)) and constant surface heat transfer coefficient (Equation (11)) seem to coincide during the early part of the temperature-time profiles. This is, of course, no surprise given that these models use more input from the experiments compared to Equation (10). The advantage of using the proposed time-dependent surface heat transfer coefficients is obviously to reach an optimum combination of good simulation of both the early temperature development and hitting the right final temperature. Figure 21 illustrates the effect different constant $\alpha$-values (Equation (11)) have on the simulated final temperature. The main advantage of using a time dependent, converging, surface heat transfer is that both the final temperature and the actual time-dependent evolution can be simulated better with our proposed model. Thus, the rather simple formula seems to be applicable when we want to simulate heat flow in materials subject to rapidly changing weather conditions or indoor temperature changes by heating- or cooling systems.

\section{Conclusions}

A simple experiment was made to study total heat surface transfer coefficients $\alpha$ in building materials over relatively short time. $\alpha$-values were determined using an experiment with very limited convection and little/no effect of phase change. Based on temperature-time measurements in varying positions in ambient air, on the material surface and in the material, $\alpha$ was modeled for varying boundary conditions. The value of $\alpha$ at different time steps based on measured temperatures were obtained with a finite different method and compared with solutions obtained using Taylor polynomials. An exponential function was adapted to describe $\alpha(t)$ and finally used in numerical simulation of temperature-time developments during rapid uni-axial cooling. The numerical simulations showed that the obtained $\alpha(t)$ - function gives results much closer to the measured temperature-time profiles than when using constant $\bar{\alpha}$-values and that the main advantage with the use of $\alpha(t)$ is the improved simulation of both final temperature and shorttime temperature development.

\section{Acknowledgements}

The authors are grateful to Dmitry Sorvanov and Svein Erik Sveen at Narvik University College, for their experimental work. The research work is part of a project performed in collaboration between Narvik University 
College and The University of Tromsø. This investigation was partly supported by the NT Programme at the research park in Tromsø, Geir Sommerhein and Eric Strand, and by the RECON programme at Norut Technology.

\section{References}

[1] D. M. Burch, D. F. Krintz and R. S. Spain, "The Effect of Wall Mass on Winter Heating, Loads and Indoor Comfort -An Experimental Study," ASHRAE Transactions, Vol. 90, No. 2, 1984.

[2] J. E. Braun, "Reducing Energy Costs and Peak Electricity Demand through Optimal Control of Building Thermal Storage," ASHRAE Transactions, Vol. 96, No. 2, 1990, pp. 876-888.

[3] F. Biasioli and M. Öberg, "Concrete for Energy Efficient and Comfortable Buildings," Proceedings of International Conference on Sustainability in the Cement and Concrete industry, In: S. Jacobsen, P. Jahren and K. Kjellsen, Eds., Norwegian Concrete Association, Liilehammer, 2007, pp. 593-599.

[4] Ø Bjøntegaard, T. Kanstad and E. J. Sellevold, "Deformation Properties and Crack Sensitivity in Young Concrete: Experience from a 4-year R\&D Project," Proceedings of Nordic Concrete Research, Vol. 33, 2005, pp. 379-381.

[5] S. Jacobsen, H. T. Nguyen and F. Melandsø, "Moisture Flow and Frost Exposure of Cement Based Materials," Proceedings of Nordic Concrete Research, Vol. 33, 2005, pp. $45-47$.

[6] S. Jacobsen, T. A. Hammer and E. J. Sellevold, "Frost testing of High Strength Concrete: Internal Cracking vs. Scaling of OPC and Silica Fume Concretes," In: S. Lindmark, Ed., "Frost Resistance of Building Materials," Proceedings of Nordic Research Seminar, Lund, 1996, pp. 49- 68.

[7] J. R. Welty, C. E. Wicks, R. E. Wilson and G. L. Rorrer, "Fundamentals of Momentum, Heat and Mass Transfer," 4th Edition, John Wiley \& Sons, Inc., New York, 2001.

[8] C. E. Hagentoft, "Introduction to Building Physics," Studentlitteratur, Lund, 2001.

[9] F. P. Incropera and D. P. DeWitt, "Fundamentals of Heat and Mass Transfer," 5th Edition, John Wiley \& Sons, Inc., 2002.
[10] R. B. Bird, W. E. Stewart and E. N. Lighfoot, "Transport Phenomena," 2nd Edition, John Wiley\& Sons, Inc., 2002.

[11] I. Lind, "Surface Heat Transfer in Thawing by Forced Air Convection," Journal of Food Engineering, Vol. 7, 1988, pp. 19-39.

[12] J. W. Baughn and S. Shimizu, "Heat Transfer Measurements from a Surface with Uniform Heat Flux and an Impinging Jet," Journal of Heat Transfer, Vol. 111, 1989, pp. 1096-1098.

[13] A. Sarkar and R. P. Singh, "Spatial Variation of Convective Heat Transfer Coefficient in Air Impingement Applications," Journal of Food Science, Vol. 68, No. 3, 2003, pp. 910-916.

[14] B. A. Anderson and R. P. Singh, "Effective Heat Transfer Coefficient Measurement during Air Impingement Thawing Using an Inverse Method," International Journal of Refrigeration, Vol. 29, 2006, pp. 281-293.

[15] H. Nguyen, S. Jacobsen and S. E. Sveen, "Modeling and Measuring Temperature Distribution in Concrete," Narvik University College, Narvik, 2005, p. 23.

[16] D. Sorvanov, "Studies of Heat Flow in Light Weight Aggregate Composites," Narvik University College, Narvik, 2005 , p. 8, 25.

[17] http://www.geofil-bubbles.com

[18] "Cement Part 1: Composition, Specifications and Conformity Criteria for Common Cements," Standard Norge ICS 91.100.10, 2005.

[19] B. Adl-Zarrabi, "Determination of Thermal Properties Og Geofil Concrete," SP. Swedish National Testing and Research Institute, Test Report P502236, 2005.

[20] A. D. Irwing, T. Dewson, G. Hong and B. Day, "Time Series Estimation of Convective Heat Transfer Coefficients," Building and Environment, Vol. 29, No. 1, 1994, pp. 89-96.

[21] I. O. Mohamed, “An Inverse Lumped Capacitance Method for Determination of Heat Transfer Coefficients for Industrial Air Blast Chillers," Food Research International, Vol. 41, No. 4, 2008, pp. 404-410.

[22] http://www.comsol.com

[23] van Schijndel, "AWM, Modelling and Solving Building Physics Problems with FemLab," Building and Environment, Vol. 38, 2003, pp. 319-327. 